\title{
AN OPTIMAL CHOICE OF DIRICHLET POLYNOMIALS FOR THE NYMAN-BEURLING CRITERION
}

\author{
S. BETTIN, J. B. CONREY, D. W. FARMER \\ In memory of Professor A. A. Karatsuba on the 75th anniversary of his birth
}

\begin{abstract}
We give a conditional result on the constant in the Báez-Duarte reformulation of the Nyman-Beurling criterion for the Riemann Hypothesis. We show that assuming the Riemann hypothesis and that $\sum_{\rho} \frac{1}{\left|\zeta^{\prime}(\rho)\right|^{2}} \ll T^{3 / 2-\delta}$, for some $\delta>0$, the value of this constant coincides with the lower bound given by Burnol.
\end{abstract}

\section{INTRODUCTION}

The Nyman-Beurling-Báez-Duarte approach to the Riemann hypothesis asserts that the Riemann hypothesis is true if and only if

$$
\lim _{N \rightarrow \infty} d_{N}^{2}=0
$$

where

$$
d_{N}^{2}=\inf _{A_{N}} \frac{1}{2 \pi} \int_{-\infty}^{\infty}\left|1-\zeta A_{N}(1 / 2+i t)\right|^{2} \frac{d t}{\frac{1}{4}+t^{2}}
$$

and the infimum is over all Dirichlet polynomials $A_{N}(s)=\sum_{n=1}^{N} \frac{a_{n}}{n^{s}}$ of length $N$ (see [Bag] for a nice account of this).

An open question is to determine what the rate of convergence of $d_{n}$ to zero is, assuming the Riemann hypothesis. Balazard and de Roton showed that, if the Riemann hypothesis is true, then

$$
d_{N}^{2} \ll \frac{(\log \log N)^{\frac{5}{2}+\varepsilon}}{\sqrt{\log N}},
$$

for all $\varepsilon>0$. On the other hand Báez-Duarte, Balazard, Landreau and Saias [BBLS00, BBLS05] showed (unconditionally) that $d_{N}^{2}$ can not decay faster than a constant times $\frac{1}{\log N}$. More precisely, they showed that

$$
\liminf _{N \rightarrow \infty} d_{N}^{2} \log N \geq \sum_{\Re(\rho)=1 / 2} \frac{1}{|\rho|^{2}},
$$

2010 Mathematics Subject Classification. Primary: 11M26. 
where here and in the following the sum is restricted to distinct zeros of the Riemann zeta function on the critical line. The constant was later improved by Burnol [Bur] who showed

$$
\liminf _{N \rightarrow \infty} d_{N}^{2} \log N \geq \sum_{\Re(\rho)=1 / 2} \frac{m(\rho)^{2}}{|\rho|^{2}},
$$

where $m(\rho)$ denotes the multiplicity of $\rho$. This lower bound is believed to be optimal and one expects that

$$
d_{N}^{2} \sim \frac{1}{\log N} \sum_{\Re(\rho)=1 / 2} \frac{m(\rho)^{2}}{|\rho|^{2}} .
$$

Notice that under the Riemann hypothesis, one has

$$
\sum_{\Re(\rho)=1 / 2} \frac{m(\rho)}{|\rho|^{2}}=2+\gamma-\log 4 \pi
$$

and in particular, if all the non-trivial zeros of $\zeta(s)$ are simple, then (1) can be rewritten as

$$
d_{N}^{2} \sim \frac{2+\gamma-\log 4 \pi}{\log N} .
$$

It is the purpose of this note to prove (1) under the Riemann Hypothesis and assuming a mild condition on the growth of the mean value of $\frac{1}{\left|\zeta^{\prime}(\rho)\right|^{2}}$ over the non-trivial zeros $|\rho| \leq T$ of $\zeta(s)$. This will be achieved by using the Dirichlet polynomial

$$
V_{N}(s):=\sum_{n=1}^{N}\left(1-\frac{\log n}{\log N}\right) \frac{\mu(n)}{n^{s}} .
$$

Theorem 1. If the Riemann hypothesis is true and if

$$
\sum_{|\Im(\rho)| \leq T} \frac{1}{\left|\zeta^{\prime}(\rho)\right|^{2}} \ll T^{\frac{3}{2}-\delta}
$$

for some $\delta>0$, then

$$
\frac{1}{2 \pi} \int_{-\infty}^{\infty}\left|1-\zeta V_{N}(1 / 2+i t)\right|^{2} \frac{d t}{\frac{1}{4}+t^{2}} \sim \frac{2+\gamma-\log 4 \pi}{\log N}
$$

The condition (2) implicitly assumes that the zeros of the Riemann zeta function are all simple. Moreover, this upper bound is "mild" in the sense that a conjecture, due to Gonek and recovered by a different heuristic method of Hughes, Keating, and O'Connell [HKO], predicts that

$$
\sum_{|\rho| \leq T} \frac{1}{\left|\zeta^{\prime}(\rho)\right|^{2}} \sim \frac{6}{\pi^{3}} T .
$$

We remark that Theorem 1 is in contrast to what one might have expected after viewing the graphs of Landreau and Richards [LR] which at first sight suggest that $V_{N}$ is not optimal. 
This behaviour of the Riemann zeta function resembles that of polynomials. In fact, Grenander and Rosenblatt [GR] (see also Theorem 2.1 in [Bur]) showed that for a polynomial $P(z)$ one has that the zeros of $P$ are all located outside or on the unit circle if and only if $\lim _{N \rightarrow \infty} \delta_{N}=0$, where

$$
\delta_{N}^{2}=\frac{1}{2 \pi} \inf _{Q_{N}} \int_{0}^{2 \pi}\left|1-P(z) Q_{N}(z)\right|^{2} \mathrm{~d} \theta,
$$

where $z=e^{i \theta}$ and the infimum is over polynomials $Q_{N}$ of degree at most $N$. Moreover, if this happens, then

$$
\lim _{N \rightarrow \infty} N \delta_{N}^{2}=\sum_{|\rho|=1} m(\rho)^{2}
$$

where the sum is restricted to the distinct zeros $\rho$ of $P(z)$ lying on the unit circle and $m(\rho)$ is again the multiplicity of $\rho$.

This analogy seems to apply also to the choices of optimal polynomials.

Theorem 2. Let $P(z)$ be a polynomial whose zeros are all simple and lie outside or on the unit circle. Let

$$
W_{N}(z):=\sum_{n=0}^{N}\left(1-\frac{n}{N}\right) a_{n} z^{n}
$$

where

$$
\frac{1}{P(z)}=\sum_{n \geq 0} a_{n} z^{n}
$$

is the Taylor expansion in $x=0$ of the inverse of $P(z)$ (i.e. it is the formal power series inverse of $P(z))$. Then

$$
\frac{1}{2 \pi} \int_{0}^{2 \pi}\left|1-P(z) W_{N}(z)\right|^{2} \mathrm{~d} \theta \sim \frac{1}{N} \sum_{|\rho|=1} m(\rho)^{2},
$$

where $z=e^{i \theta}$.

We remark that the proofs of Theorem 1 and 2 are very similar, the main difference being that the Riemann zeta function has infinitely many zeros. This generates some issues concerning the convergence of certain sums of $\frac{1}{\zeta^{\prime}(\rho)}$, which force us to assume condition (2).

\section{Polynomials}

Lemma 1. Let $P(s)$ be a polynomial with $P(0) \neq 0$. We have

$$
W_{N}(s)=\frac{1}{P(s)}\left(1+\frac{s}{N} \frac{P^{\prime}}{P}(s)\right)-\frac{s}{N} Y_{N}(s),
$$


where $W_{N}(s)$ is defined in (3),

$$
Y_{N}(s):=\sum_{\rho} \operatorname{Res}_{z=\rho} \frac{s^{N}}{P(z)(z-s)^{2} z^{N}}
$$

and the sum is over distinct zeros $\rho$ of $P(z)$.

Proof. Since $P(0) \neq 0$, we can take an $\varepsilon>0$ such that all the zeros of $P(z)$ lie outside of the circle $|z|=\varepsilon$. Now, observe that we can assume $0<|s|<\varepsilon$, since the result will then extend to all $\mathbb{C}$ by analytic continuation. Denoting by $\mathcal{C}_{y}$ the circle of radius $y>0$ (oriented in the positive direction), by the residue theorem we have that

$$
a_{n}=\frac{1}{2 \pi i} \int_{\mathcal{C}_{\varepsilon}} \frac{1}{P(z)} \frac{\mathrm{d} z}{z^{n+1}}
$$

therefore

$$
W_{N}(s)=\frac{1}{2 \pi i} \int_{\mathcal{C}_{\varepsilon}} \frac{1}{P(z)} \sum_{n=0}^{N}\left(1-\frac{n}{N}\right)\left(\frac{s}{z}\right)^{n} \frac{\mathrm{d} z}{z} .
$$

Now,

$$
\sum_{n=0}^{N}\left(1-\frac{n}{N}\right) z^{n}=-\frac{1}{N} \frac{z-z^{N+1}}{(1-z)^{2}}+\frac{1}{1-z}
$$

and thus

$$
W_{N}(s)=\frac{1}{2 \pi i} \int_{\mathcal{C}_{\varepsilon}} \frac{1}{P(z)}\left(-\frac{1}{N} \frac{s z^{N}-s^{N+1}}{(z-s)^{2} z^{N}}+\frac{1}{z-s}\right) \mathrm{d} z .
$$

Now, by the residue theorem

$$
\frac{1}{2 \pi i} \int_{\mathcal{C}_{\varepsilon}} \frac{1}{P(z)}\left(-\frac{1}{N} \frac{s}{(z-s)^{2}}+\frac{1}{z-s}\right) \mathrm{d} z=\frac{1}{P(s)}\left(1+\frac{s}{N} \frac{P^{\prime}(s)}{P(s)}\right),
$$

whereas, moving the line of integration to $\mathcal{C}_{y}$ and letting $y$ tend to infinity, one has that

$$
\frac{1}{2 \pi i N} \int_{\mathcal{C}_{\varepsilon}} \frac{1}{P(z)} \frac{s^{N+1}}{(z-s)^{2} z^{N}} \mathrm{~d} z=-\frac{s}{N} Y_{N}(s)
$$

and the Lemma follows.

Proof of Theorem 2. Let $\delta>1$ be such that $P(s)$ does not have any zero on $1<|s| \leq \delta$. We have

$$
\begin{aligned}
\frac{1}{2 \pi} \int_{0}^{2 \pi}\left|1-P(z) W_{N}(z)\right|^{2} \mathrm{~d} \theta & =\frac{1}{2 \pi i} \int_{\mathcal{C}_{1}}\left(1-P(s) W_{N}(s)\right)\left(1-\bar{P}\left(\frac{1}{s}\right) \bar{W}_{N}\left(\frac{1}{s}\right)\right) \frac{\mathrm{d} s}{s} \\
& =\frac{1}{2 \pi i} \int_{\mathcal{C}_{\delta}}\left(1-P(s) W_{N}(s)\right)\left(1-\bar{P}\left(\frac{1}{s}\right) \bar{W}_{N}\left(\frac{1}{s}\right)\right) \frac{\mathrm{d} s}{s} .
\end{aligned}
$$


Therefore, by Lemma 1, this is

$$
\frac{1}{2 \pi i N^{2}} \int_{\mathcal{C}_{\delta}}\left(\frac{P^{\prime}}{P}(s)-P(s) Y_{N}(s)\right)\left(\frac{\bar{P}^{\prime}}{\bar{P}}\left(\frac{1}{s}\right)-\bar{P}\left(\frac{1}{s}\right) \bar{Y}_{N}\left(\frac{1}{s}\right)\right) \frac{\mathrm{d} s}{s} .
$$

Now, for $|s|=\delta$ one has

$$
Y_{N}(s) \bar{Y}_{N}\left(\frac{1}{s}\right)=O(1)
$$

therefore

$$
\frac{1}{2 \pi i N^{2}} \int_{\mathcal{C}_{\delta}}\left(\frac{P^{\prime}}{P}(s) \frac{\bar{P}^{\prime}}{\bar{P}}\left(\frac{1}{s}\right)+P(s) Y_{N}(s) \bar{P}\left(\frac{1}{s}\right) \bar{Y}_{N}\left(\frac{1}{s}\right)\right) \frac{\mathrm{d} s}{s}=O\left(\frac{1}{N^{2}}\right) .
$$

Moreover for $s \in \mathcal{C}_{\delta}$ one has that $\bar{Y}_{N}\left(\frac{1}{s}\right)=O\left(\delta^{-N}\right)$, thus

$$
-\frac{1}{2 \pi i N^{2}} \int_{\mathcal{C}_{\delta}}\left(\frac{P^{\prime}}{P}(s) \bar{P}\left(\frac{1}{s}\right) \bar{Y}_{N}\left(\frac{1}{s}\right)\right) \frac{\mathrm{d} s}{s}=O\left(\delta^{-N} / N^{2}\right) .
$$

Finally, by the residue theorem,

$$
\begin{aligned}
-\frac{1}{2 \pi i N^{2}} \int_{\mathcal{C}_{\delta}} P(s) Y_{N}(s) \frac{\bar{P}^{\prime}}{\bar{P}}\left(\frac{1}{s}\right) \frac{\mathrm{d} s}{s}= \\
=-\frac{1}{N^{2}} \sum_{|\rho|=1} \operatorname{Res}_{s=\rho} P(s) Y_{N}(s) \frac{\bar{P}^{\prime}}{\bar{P}}\left(\frac{1}{s}\right) \frac{1}{s}+ \\
-\frac{1}{2 \pi i N^{2}} \int_{\mathcal{C}_{\frac{1}{\delta}}} P(s) Y_{N}(s) \frac{\bar{P}^{\prime}}{\bar{P}}\left(\frac{1}{s}\right) \frac{\mathrm{d} s}{s} \\
=-\frac{1}{N^{2}} \sum_{|\rho|=1} \operatorname{Res}_{s=\rho} P(s) Y_{N}(s) \frac{\bar{P}^{\prime}}{\bar{P}}\left(\frac{1}{s}\right)+O\left(\delta^{-N} / N^{2}\right) .
\end{aligned}
$$

The theorem then follows by observing that

$$
\operatorname{Res}_{s=\rho} P(s) Y_{N}(s) \frac{\bar{P}^{\prime}}{\bar{P}}\left(\frac{1}{s}\right) \frac{1}{s}=-N+O(1) .
$$

\section{The Riemann Zeta-Function}

We start with the following lemma, which is the analogue of Lemma 1. We remark that this lemma is unconditional. 
Lemma 2. If $0<\Re(s)<1$, then

$$
V_{N}(s)=\frac{1}{\zeta(s)}\left(1-\frac{1}{\log N} \frac{\zeta^{\prime}}{\zeta}(s)\right)+\frac{1}{\log N} \sum_{\rho} R_{N}(\rho, s)+\frac{1}{\log N} F_{s}(1 / N),
$$

where the sum is over distinct non-trivial zeros $\rho$ of $\zeta(s)$ with

$$
R_{N}(\rho, s)=\operatorname{Res}_{z=\rho} \frac{N^{z-s}}{\zeta(z)(z-s)^{2}}
$$

and where

$$
F_{s}(z)=\pi z^{s} \sum_{n=1}^{\infty} \frac{(-1)^{n}(2 \pi)^{2 n+1} z^{2 n}}{(2 n) ! \zeta(2 n+1)(2 n+s)^{2}}
$$

is an entire function of $z$.

Proof. We have

$$
V_{N}(s)=\frac{1}{\log N} \frac{1}{2 \pi i} \int_{(2)} \frac{N^{w}}{\zeta(s+w)} \frac{d w}{w^{2}},
$$

where we use the notation $\int_{(c)}$ to mean an integration up the vertical line from $c-i \infty$ to $c+i \infty$. Now we move the path of integration to $\Re(w)=-\Re(s)-2 M-1$ for a large integer $M$. The residue at $w=\rho-s$ is $R_{N}(\rho, s) / \log N$. The residue at $s+w=-2 n$ is

$$
\frac{N^{-2 n-s}}{\zeta^{\prime}(-2 n)(2 n+s)^{2} \log N}
$$

and the integral on the new path is $\ll N^{-2 M-1}$. Letting $M \rightarrow \infty$ and using

$$
\zeta^{\prime}(-2 n)=\frac{(-1)^{n} \pi(2 n) ! \zeta(2 n+1)}{(2 \pi)^{2 n+1}}
$$

we obtain the result.

Lemma 3. Let $\varepsilon>0$. Assume the Riemann hypothesis and that all the zeros of $\zeta(s)$ are simple. Then, if condition (2) holds, for $\Re(s)=\frac{1}{2} \pm \varepsilon$ one has

$$
\sum_{\rho} R_{N}(\rho, s) \ll N^{\mp \varepsilon}|s|^{\frac{3}{4}-\frac{\delta}{2}+\varepsilon} .
$$

Proof. Firstly observe that, by the Cauchy-Schwartz inequality, (2) implies

$$
\sum_{|\rho| \leq T} \frac{1}{\left|\zeta^{\prime}(\rho)\right|} \ll \sqrt{N(T) \sum_{|\rho| \leq T} \frac{1}{\left|\zeta^{\prime}(\rho)\right|^{2}}} \ll T^{\frac{5}{4}-\frac{\delta}{2}} \sqrt{\log T}
$$

since

$$
N(T):=\frac{1}{2} \sum_{|\rho| \leq T} 1=\frac{T}{2 \pi} \log \frac{T}{2 \pi e}+O(\log T) .
$$


Therefore, by partial summation, we have that the series

$$
\sum_{\rho} \frac{1}{\left|\zeta^{\prime}(\rho)\right||\rho|^{\alpha}}
$$

is convergent for any $\alpha>\frac{5}{4}-\frac{\delta}{2}$. Now, for a simple zero $\rho$, we have

$$
R_{N}(\rho, s)=\sum_{\rho} \frac{N^{\rho-s}}{\zeta^{\prime}(\rho)(\rho-s)^{2}} .
$$

Therefore

$$
\begin{aligned}
N^{ \pm \varepsilon} \sum_{\rho} R_{N}(\rho, s) & \ll \sum_{|\rho-s|<\frac{|\rho|}{2}} \frac{1}{\left|\zeta^{\prime}(\rho)\right||\rho-s|^{2}}+\sum_{|\rho-s| \geq \frac{|\rho|}{2}} \frac{1}{\left|\zeta^{\prime}(\rho)\right||\rho-s|^{2}} \\
& \ll \sum_{|\rho-s|<\frac{|\rho|}{2}} \frac{1}{\left|\zeta^{\prime}(\rho)\right||\rho-s|^{2}}+\sum_{|\rho-s| \geq \frac{|\rho|}{2}} \frac{1}{\left|\zeta^{\prime}(\rho)\right||\rho|^{2}} \\
& \ll \sum_{|\rho-s|<\frac{|\rho|}{2}} \frac{1}{\left|\zeta^{\prime}(\rho)\right||\rho-s|^{2}}+1 .
\end{aligned}
$$

Now, by the Cauchy-Schwartz inequality,

$$
\sum_{|\rho-s|<\frac{|\rho|}{2}} \frac{1}{\left|\zeta^{\prime}(\rho)\right||\rho-s|^{2}} \ll \sqrt{\left(\sum_{|\rho|<2|s|} \frac{1}{\left|\zeta^{\prime}(\rho)\right|^{2}}\right)\left(\sum_{|\rho|<2|s|} \frac{1}{|\rho-s|^{4}}\right)} \ll|s|^{\frac{3}{4}-\frac{\delta}{2}+\varepsilon}
$$

since, by partial summation,

$$
\sum_{|\rho|<2|s|} \frac{1}{|\rho-s|^{4}} \ll \log (|s|+2) .
$$

This completes the proof of the lemma.

Proof of Theorem 1. We have

$$
\begin{aligned}
\frac{1}{2 \pi} \int_{-\infty}^{\infty} \mid & -\left.\zeta V_{N}(1 / 2+i t)\right|^{2} \frac{d t}{1 / 4+t^{2}} \\
= & \frac{1}{2 \pi i} \int_{\left(\frac{1}{2}\right)}\left(1-\zeta V_{N}(s)\right)\left(1-\zeta V_{N}(1-s)\right) \frac{d s}{s(1-s)} \\
= & \frac{1}{2 \pi i} \int_{\left(\frac{1}{2}-\varepsilon\right)}\left(1-\zeta V_{N}(s)\right)\left(1-\zeta V_{N}(1-s)\right) \frac{d s}{s(1-s)}
\end{aligned}
$$


By Lemma 2, this is

$$
\begin{aligned}
\frac{1}{\log ^{2} N} \frac{1}{2 \pi i} \int_{\left(\frac{1}{2}-\varepsilon\right)} & \left(\frac{\zeta^{\prime}}{\zeta^{2}}(s)-\sum_{\rho} R_{N}(\rho, s)-F_{s}\left(\frac{1}{N}\right)\right) \times \\
& \times\left(\frac{\zeta^{\prime}}{\zeta^{2}}(1-s)-\sum_{\rho} R_{N}(\rho, 1-s)-F_{1-s}\left(\frac{1}{N}\right)\right) \frac{\zeta(s) \zeta(1-s)}{s(1-s)} d s .
\end{aligned}
$$

Now, we have

$$
\begin{aligned}
\frac{1}{\log ^{2} N} \frac{1}{2 \pi i} & \int_{\left(\frac{1}{2}-\varepsilon\right)} \sum_{\rho_{1}, \rho_{2}} R_{N}\left(\rho_{1}, s\right) R_{N}\left(\rho_{2}, 1-s\right) \frac{\zeta(s) \zeta(1-s)}{s(1-s)} d s \\
& \ll \frac{1}{\log ^{2} N} \int_{\left(\frac{1}{2}-\varepsilon\right)} \sum_{|\rho-s|<\frac{|\rho|}{2}} \frac{1}{\left|\zeta^{\prime}(\rho)\right||\rho-s|^{2}} \frac{|d s|}{|s|^{\frac{5}{4}+\frac{\delta}{2}-5 \varepsilon}}+O\left(\frac{1}{\log ^{2} N}\right),
\end{aligned}
$$

where we used (4), (5) and the bound $\zeta\left(\frac{1}{2} \pm \varepsilon \pm i t\right) \ll|t|^{2 \varepsilon}$ (which is a consequence of the Lindelöf hypothesis). Reversing the order of summation and integration, we have that this is bounded by

$$
\begin{aligned}
\frac{1}{\log ^{2} N} \sum_{\rho} \frac{1}{\left|\zeta^{\prime}(\rho)\right|} & \int_{\left(\frac{1}{2}-\varepsilon\right)+i\left(\Im(\rho)-\frac{|\rho|}{2}\right)}^{\left(\frac{1}{2}-\varepsilon\right)+i\left(\Im(\rho)+\frac{|\rho|}{2}\right)} \frac{|d s|}{|\rho-s|^{2}|s|^{\frac{5}{4}+\frac{\delta}{2}-5 \varepsilon}}+O\left(\frac{1}{\log ^{2} N}\right) \\
& \ll \frac{1}{\log ^{2} N} \sum_{\rho} \frac{1}{\left|\zeta^{\prime}(\rho)\right||\rho|^{\frac{5}{4}+\frac{\delta}{2}-5 \varepsilon}} \ll \frac{1}{\log ^{2} N},
\end{aligned}
$$

if $\varepsilon<\frac{\delta}{10}$.

Now, by Lemma 3 and the trivial estimate $F_{s}(z)=O\left(N^{-\frac{5}{2}}\right)$, all the other terms in (6) are trivially $O\left(\frac{1}{\log ^{2} N}\right)$ apart from

$$
-\frac{1}{\log ^{2} N} \frac{1}{2 \pi i} \int_{\left(\frac{1}{2}-\varepsilon\right)} \frac{\zeta^{\prime}}{\zeta}(1-s) \sum_{\rho} R_{N}(\rho, s) \frac{\zeta(s)}{s(1-s)} d s .
$$

The integrand has a double pole at every zero $\rho$ of residue

$$
\begin{aligned}
\operatorname{Res}_{s=\rho}\left(\frac{\zeta^{\prime}}{\zeta}(1-s) \sum_{\rho} R_{N}(\rho, s) \frac{\zeta(s)}{s(1-s)}\right) & =\frac{\log N-\frac{1}{2} \frac{\zeta^{\prime \prime}(\rho)}{\zeta^{\prime}(\rho)}+\frac{\chi^{\prime}}{\chi}(\rho)+\frac{1-2 \rho}{|\rho|^{2}}}{|\rho|^{2}} \\
& =\frac{\log N}{|\rho|^{2}}+O\left(\frac{1}{|\rho|^{2-\varepsilon}\left|\zeta^{\prime}(\rho)\right|}+\frac{1}{|\rho|^{2}}\right)
\end{aligned}
$$

where we used the bound $\zeta^{\prime \prime}\left(\frac{1}{2}+i t\right) \ll|t|^{\varepsilon}$, which follows from the Lindelöf hypothesis and Cauchy's estimate for the derivatives of a holomorphic function. It follows that moving the 
line of integration in $(7)$ to $\Re(s)=\frac{1}{2}+\varepsilon$ we get that the integral is equal to

$$
\frac{1}{\log N} \sum_{\rho} \frac{1}{|\rho|^{2}}+O\left(\frac{1}{\log ^{2} N}\right),
$$

and Theorem 1 then follows.

\section{REFERENCES}

[BBLS00] Baéz-Duarte, L.; Balazard, M.; Landreau, B.; Saias, E. Notes sur la fonction $\zeta$ de Riemann. III. (French) [Notes on the Riemann $\zeta$-function. III] Adv. Math. 149 (2000), no. 1, 130-144.

[BBLS05] Baéz-Duarte, L.; Balazard, M.; Landreau, B.; Saias, E. Étude de l'autocorrelation multiplicative de la fonction 'partie fractionnaire'. (French) [Study of the multiplicative autocorrelation of the fractional part function] Ramanujan J. 9 (2005), no. 1-2, 215-240; arxiv math.NT/0306251.

[Bag] Bagchi, Bhaskar. On Nyman, Beurling and Baez-Duarte's Hilbert space reformulation of the Riemann hypothesis. Proc. Indian Acad. Sci. Math. 116 (2006), no. 2, 137-146; arxiv math.NT/0607733.

[Bur] Burnol, J.F. A lower bound in an approximation problem involving the zeros of the Riemann zeta function. Advances in Math. 170 (2002), 56-70.

[GR] Grenander, U.; Rosenblatt, M. An extension of a theorem of G. Szegö and its application to the study of stochastic processes. Trans. Amer. Math. Soc. 76, (1954), 112-126.

[HKO] Hughes, C.P.; Keating, J.P.; O'Connell, N. Random matrix theory and the derivative of the Riemann zeta function. R. Soc. Lond. Proc. Ser. A Math. Phys. Eng. Sci. 456 (2000), no. 2003, 2611-2627.

[LR] Landreau, B.; Richard, F. Le critère de Beurling et Nyman pour l'hypothèse de Riemann: aspects numériques. (French) [The Beurling-Nyman criterion for the Riemann hypothesis: numerical aspects] Experiment. Math. 11 (2002), no.3, 349-360.

Sandro Bettin - School of Mathematics, University of Bristol, Queens Ave, Bristol, BS8 $1 \mathrm{SN}, \mathrm{UK}$

Current address: Centre de Recherches Mathématiques - Université de Montral, P.O. Box 6128, Centreville Station, Montréal (Québec), H3C 3J7, Canada

J. Brian Conrey - American Institute of Mathematics, 360 Portage Avenue, Palo Alto, CA 94306 - 2244 USA

David W. Farmer - American Institute of Mathematics, 360 Portage Avenue, Palo Alto, CA 94306 - 2244 USA 